\title{
Numerical Simulations of the Interaction Between a Settling Particle and a Rising Microbubble
}

\author{
Henrik Ström ${ }^{\mathrm{a}}$, Ebrahim Karimi Sibaki ${ }^{\mathrm{b}}$ and Srdjan Sasic ${ }^{\mathrm{a}}$ \\ ${ }^{a}$ Division of Fluid Dynamics, Department of Applied Mechanics \\ ${ }^{b}$ Division of Chemical Engineering, Department of Chemical and Biological Engineering \\ Chalmers University of Technology, SE-412 96 Göteborg, Sweden
}

\begin{abstract}
In the current work the hydrodynamic interaction between a settling particle and a rising microbubble is investigated using numerical simulations. The simulations are performed in a multiphase direct numerical simulation (DNS) framework, indicating that all relevant spatial and temporal scales are resolved. It is shown that the method predicts that particle-bubble attachment is possible when the initial horizontal distance between their centers is small and that the particle will pass the bubble without attaching when this initial distance is large. Furthermore, it is shown that the probability of a successful attachment is lower if the bubble Eötvös and Morton numbers are significantly larger than unity.
\end{abstract}

Keywords: multiphase flow, Volume of Fluid (VOF), bubble-particle interaction.

PACS: 47.55.-t, 47.55.dd, 47.11.-j, 47.15.G-

\section{INTRODUCTION}

Multiphase flows with a simultaneous presence of both particles and bubbles suspended in a liquid are important in a great number of industrial processes. For example, bubbles are used within separation processes such as flotation and antifoaming, in which bubble-particle interaction is a key phenomenon [1]. The aim is to make particles attach to bubbles so that they rise together through a suspension. These agglomerates can then be skimmed off from the open surface of the suspension [2]. Froth flotation, ion flotation, foam fractionation, waste water treatment, and foam separation of proteins are several examples of such processes in which millions of tons of material are treated annually [3]. A fundamental step toward understanding of these processes is the investigation of bubble-particle interactions, which is the topic of the present work.

The initial horizontal distance between centres of gravity of a bubble and a particle, when they are still far to interact, is denoted the separation distance. Successful attachment of a particle to a bubble depends on the separation distance, bubble size, particle density, hydrophobic or hydrophilic properties of the particle surface, as well as the forces between the bubble and the particle [4]. Of these forces, some (the Van der Waals and electrostatic forces) become significant only when the distance between bubble surface and particle surface is very small, typically below $100 \mathrm{~nm}$ [3]. In this paper, we therefore limit ourselves to the drag, gravity and buoyancy forces and perform numerical simulations resolving the interaction between a rising microbubble $\left(d_{p}=130 \mu \mathrm{m}\right)$ and a settling solid particle $\left(d_{p}=50 \mu \mathrm{m}\right)$. A suitable framework for conducting the simulations is the Volume of Fluid (VOF) multiphase model. The simulation framework has no restriction on relative sizes of a bubble or a solid particle. The advantage of the method is that the shape and trajectory of a bubble and also the trajectory of a particle can be tracked throughout the interactions.

\section{NUMERICAL METHOD}

We consider here a three-phase flow problem: water as surrounding fluid, an air bubble as gas phase, and a latex particle as the solid phase. The VOF method was originally proposed by Hirt and Nichols [5] for simulation of gasliquid and liquid-liquid systems. Using VOF for handling also solid particles motion requires some additional considerations and assumptions, as discussed by Ström et al. [6]. The same method is used in the present work.

The volume fraction of the $i^{\text {th }}$ phase in each computational cell is denoted as $\alpha_{i}$ ( $i=1$ (liquid), 2 (bubble) or 3 (particle)). For the cells only filled with phase $1, \alpha_{1}$ is equal to unity and for the cells occupied only by the second or

Numerical Analysis and Applied Mathematics ICNAAM 2012

AIP Conf. Proc. 1479, 113-116 (2012); doi: 10.1063/1.4756075

(C) 2012 American Institute of Physics 978-0-7354-1091-6/\$30.00 
the third phase, $\alpha_{1}$ equals zero. If a cell is filled by two or three phases, the average value is used for the density $(\rho)$ and viscosity $(\mu)$ :

$$
\begin{aligned}
& \rho=\sum_{i=1}^{3} \alpha_{i} \rho_{i} \\
& \mu=\sum_{i=1}^{3} \alpha_{i} \mu_{i}
\end{aligned}
$$

In theory, the viscosity of the solid particle should approach infinity. It can be shown, however, that satisfactory results can be obtained by employing a sufficiently high numerical value instead (several orders of magnitude larger than that of the continuous phase) [6].

The incompressible continuity and momentum equations are used to determine the velocity field $u$ :

$$
\begin{gathered}
\nabla \cdot u=0 \\
\rho\left[\frac{\partial u}{\partial t}+u \cdot \nabla u\right]=-\nabla P+\mu \nabla^{2} u+\rho g+F_{\sigma}
\end{gathered}
$$

where $u$ is the velocity, $P$ is the pressure, $g$ is the gravitational acceleration and $F_{\sigma}$ is the surface tension force. The latter is calculated using the continuum surface tension force model of Brackbill et al. [7]. The concept of surface tension has no direct physical interpretation for the interface between the water and the solid particle. The ability of the surface tension force model to counteract particle deformation is however taken advantage of in the current framework [6]. More specifically, an artificial water-particle surface tension is continuously adjusted to ensure that the Capillary number $(\mathrm{Ca}=\mu u / \sigma)$ remains at or below 0.1 throughout a simulation.

The interface between the phases is tracked by solving the advection equation for the volume fraction of the phases in each computational cell:

$$
\frac{\partial \alpha_{i}}{\partial t}+\nabla \cdot\left(\alpha_{i} u\right)=0
$$

The equations (3-4) are solved in a finite volume framework using a pressure-based, segregated solver on a colocated grid. The pressure-velocity coupling is handled using the PISO scheme and the pressure interpolation scheme used is PRESTO!. Equation (5) is discretized using the CICSAM scheme.

\section{RESULTS AND DISCUSSION}

We first validate our computational method by comparing the results to experimental data [8]. Then, we examine the interaction between a solid particle and a bubble by investigating the influence of the separation distance and the ability of the bubble to deform on the particle and bubble trajectories.

\section{Validation}

The VOF method is known to produce predictions of bubble motion in good agreement with the available experimental data [9]. As the application of VOF-based methods to simulations of solid particles motion is a newer field, it is important to validate the predictions of solid particle interactions against experimental data. Adamczyk et al. [8] studied the approach of a particle to another one along their line of centers. They found that the drag on such an interactive particle is larger than the Stokes drag by a factor $\lambda$, referred to as the drag correction function.

To validate the current VOF method, the motion of a solid particle towards a stationary particle of identical size is simulated, and the predicted drag correction function is extracted and plotted versus the normalized distance 
between the two particles. This normalized distance is taken as the interparticle distance, excluding both radii, divided by the particle radius. Figure 1 shows that the predicted drag correction function from the proposed method is in very good agreement with the experimental data of Adamczyk et al. [8].

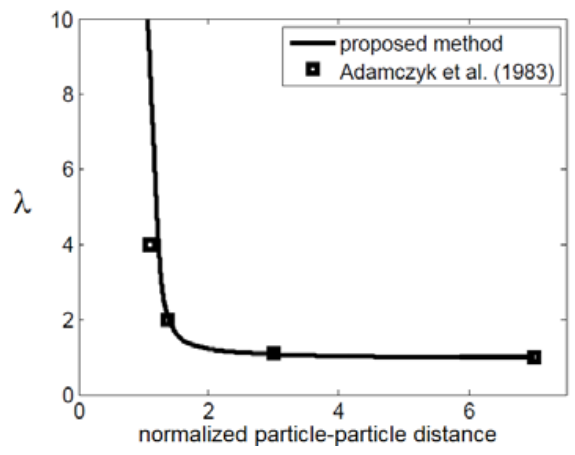

FIGURE 1. The particle drag correction function $(\lambda)$ plotted as a function of the normalized particle-particle distance.

\section{Bubble-particle interaction}

A number of different scenarios are possible in the interaction between a settling particle and a rising bubble. The first cases here deal with the situation when the Reynolds and Eötvös numbers are about unity or smaller, so that the bubble remains spherical and within the Stokes regime, as is typical for microbubbles [10].

The initial separation distance is a parameter of great interest, due to the existence of a critical separation distance (often called the grazing radius). For a separation distance smaller than the grazing radius, the particle and bubble always collide. In contrast, at separation distances larger than the grazing radius, no collision occurs and the particle passes the bubble [11]. If the bubble and the particle are initially on a collision course, as in an axisymmetric configuration (not shown here), the outcome of the interaction is determined by the buoyancy of the bubble and particle combined. For the combination of particle and bubble investigated in the current work, the air bubble is large enough for the particle to slow down and be carried upwards by the bubble. Eventually, the particle will attach to the bubble.

When the particle and the bubble are initially separated by a distance equal to the bubble radius in the horizontal direction, they avoid each other due to the hydrodynamic interaction, as shown in Figure 2.
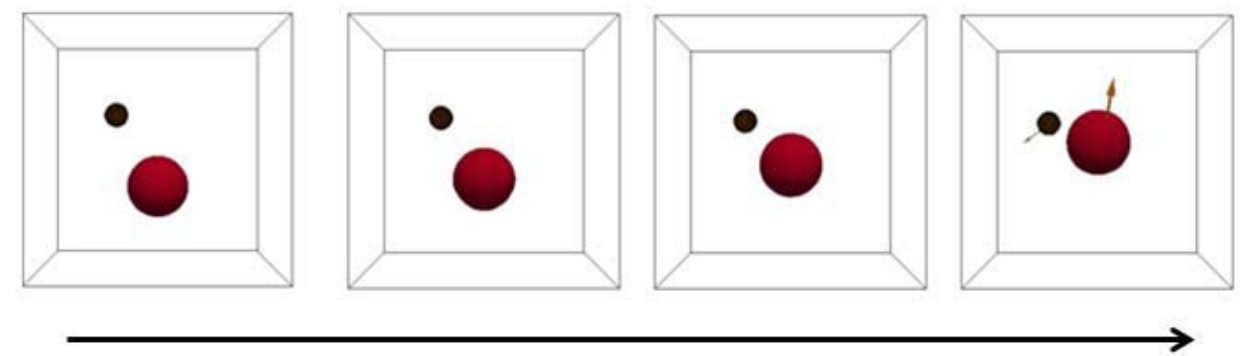

FIGURE 2. Interaction between an air bubble (red) and a latex particle (black) in water. The long black arrow indicates the direction of increasing time. When the settling particle is initially further than a radius away from the bubble center of motion, the bubble and particle do not collide, but avoid each other.

The third and fourth cases illustrate the change in the bubble-particle interaction that can occur when the bubble is deforming. This implies that the Eötvös and Morton numbers are several orders of magnitude larger than in the first two cases. Physically, this happens when the bubble is significantly larger than the microbubble considered here and/or the water contains highly surface-active components. In the present work, the aim is primarily to demonstrate the capabilities of the computational framework. Therefore, this behavior is provoked numerically by decreasing the 
surface tension between the air and the water. In this way, all other parameters - such as the particle-to-bubble size ratio - can be kept constant for the purpose of the comparison.

In these two cases, the initial horizontal separation between the particle and the bubble is chosen to be approximately equal to the grazing radius (as predicted by the Gaudin model [12]). As shown in the upper part of Figure 3, the non-deforming bubble comes so close to the particle that they are likely to attach, as is expected for this initial configuration. However, as is shown in the lower section of Figure 3, under circumstances that allow bubble deformation, the collision is avoided.

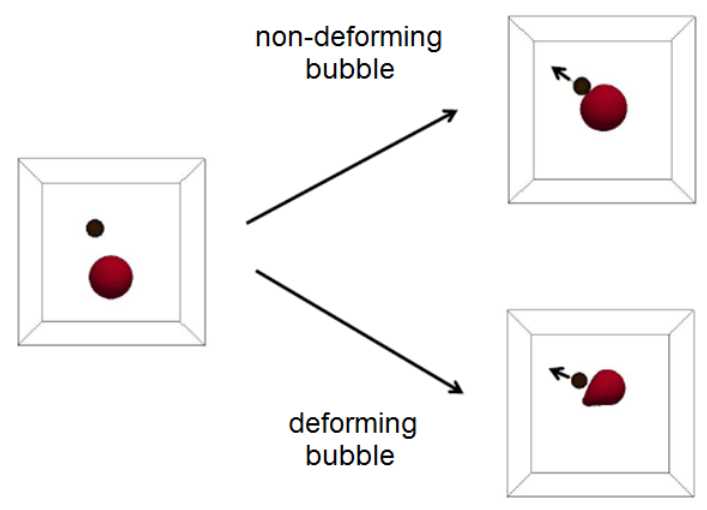

FIGURE 3. Interaction between an air bubble (red) and a latex particle (black) in water. The initial separation between the two centers of motion is smaller than the bubble radius, the bubble and particle collide if the bubble is non-deforming. However, if the Eötvös and Morton numbers are large, the bubble will deform which prevents the collision.

\section{CONCLUSIONS}

In the current work, the interaction between a settling particle $\left(d_{p}=50 \mu \mathrm{m}\right)$ and a rising microbubble $\left(d_{p}=130\right.$ $\mu \mathrm{m})$ is investigated using numerical simulations. The simulations are performed in a multiphase direct numerical simulation framework, indicating that all relevant spatial and temporal scales are resolved. The framework chosen is the Volume of Fluid (VOF) multiphase model, which has been extended to allow handling of motion of solid particles.

It is shown that the method predicts the interaction between two solid particles in good agreement with the available experimental data [8]. Furthermore, the method predicts that particle-bubble attachment is possible when the initial horizontal distance is smaller than the grazing radius, and that the particle will pass by the bubble without attachment when this initial distance is significantly larger than the grazing radius. Finally, it is also shown that the probability of a successful attachment is lower if the bubble Eötvös and Morton numbers are significantly larger than unity.

\section{REFERENCES}

1. F. Omota, A. C. Dimian and A. Bliek, Chem. Eng. Sci.61, 835-844 (2006).

2. B. Albijanic, O. Ozdemir, A. V. Nguyen and D. Bradshaw, Adv. Colloid Interface Sci. 159, 1-21 (2010).

3. K. Malysa, M. Krasowska and M. Krzan, Adv. Colloid Interface Sci. 114-115, 205-225 (2005).

4. X. Fan, Z. Zhang, G. Li and N. A. Rowson, Chem. Eng. Sci. 59, 2639-2645 (2004).

5. C. W. Hirt and B. D. Nichols, J. Comput. Phys. 39, 201-225 (1981).

6. H. Ström, S. Sasic and B. Andersson, Int. J. Multiphase Flow 37, 906-918 (2011).

7. J. U. Brackbill, D. B. Kothe and C. Zemach, J. Comput. Phys. 100, 335-354 (1992).

8. Z. Adamczyk, M. Adamczyk and T. G. M. van de Ven, J. Colloid Interface Sci. 96, 204-213 (1983).

9. F. Raymond and J.-M. Rosant, Chem. Eng. Sci. 55, 943-955 (2000).

10. R. Clift, J. R. Grace and M. E. Weber, Bubbles, Drops, and Particles, New York: Academic Press, Inc., 1978.

11. A. Nguyen Van and S. Kmet, Int. J. Mineral Proc. 35, 205-223 (1992).

12. A. M. Gaudin, Flotation, $2^{\text {nd }}$ edition, New York: McGraw-Hill, 1957. 